\title{
Neutrophil extracellular traps in the host defense against sepsis induced by Burkholderia pseudomallei (melioidosis)
}

Hanna K de Jong ${ }^{1,2^{*}}$, Gavin CKW Koh' ${ }^{3,4,5}$, Ahmed Achouiti ${ }^{1}$, Anne J van der Meer ${ }^{1}$, Ingrid Bulder ${ }^{6}$, Femke Stephan ${ }^{6}$, Joris JTH Roelofs ${ }^{7}$, Nick PJ Day ${ }^{4,8}$, Sharon J Peacock ${ }^{3,4}$, Sacha Zeerleder ${ }^{6,9}$ and W Joost Wiersinga ${ }^{1,2}$

* Correspondence:
h.k.dejong@amc.uva.nl
${ }^{1}$ Center for Experimental and
Molecular Medicine (CEMM),
Academic Medical Center,
Meibergdreef 9, Room G2-132,
Amsterdam 1105 AZ, The
Netherlands
Department of Medicine, Division
of infectious diseases, Academic
Medical Center, Meibergdreef 9,
Room G2-132, Amsterdam 1105 AZ,
The Netherlands
Full list of author information is
available at the end of the article

available at the end of the article

\begin{abstract}
Background: Neutrophil extracellular traps (NETs) are a central player in the host response to bacteria: neutrophils release extracellular DNA (nucleosomes) and neutrophil elastase to entrap and kill bacteria. We studied the role of NETs in Burkholderia pseudomallei infection (melioidosis), an important cause of Gram-negative sepsis in Southeast Asia.
\end{abstract}

Methods: In a prospective observational study, circulating nucleosomes and neutrophil elastase were assayed in 44 patients with Gram-negative sepsis caused by B. pseudomallei (melioidosis) and 82 controls. Functional assays included human neutrophil stimulation and killing assays and a murine model of B. pseudomallei infection in which NET function was compromised using DNase. Specified pathogen-free 8- to 12-week-old C57BL/6 mice were sacrificed post-infection to assess bacterial loads, inflammation, and pathology.

Results: Nucleosome and neutrophil elastase levels were markedly elevated in patients compared to controls. NETs killed B. pseudomallei effectively, and neutrophils stimulated with B. pseudomallei showed increased elastase and DNA release in a time- and dose-dependent matter. In mice, NET disruption with intravenous DNase administration resulted in decreased nucleosome levels. Although DNase treatment of mice resulted in diminished liver inflammation, no differences were observed in bacterial dissemination or systemic inflammation.

Conclusion: B. pseudomallei is a potent inducer of NETosis which was reflected by greatly increased levels of NET-related components in melioidosis patients. Although NETs exhibited antibacterial activity against B. pseudomallei, NET formation did not protect against bacterial dissemination and inflammation during B. pseudomallei-induced sepsis.

Keywords: Sepsis; Burkholderia pseudomallei; Melioidosis; Neutrophil extracellular traps (NETs); Neutrophils; Diabetes; Survival; DNase; Innate immunity

\section{Background}

Melioidosis (Gram-negative infection caused by Burkholderia pseudomallei) is a major cause of severe community-acquired sepsis in Southeast Asia and northern Australia $[1,2]$. The clinical manifestations of B. pseudomallei infection range from chronic skin abscess to acute fulminant sepsis. Despite antibiotic treatment, melioidosis patients with bacteremia or pneumonia have a mortality rate of approximately 40\% [3,1,2]. Melioidosis is therefore a good clinical model in which to study Gram-negative sepsis [4-6].

\section{Springer}

(c) 2014 de Jong et al.; licensee Springer. This is an Open Access article distributed under the terms of the Creative Commons Attribution License (http://creativecommons.org/licenses/by/2.0), which permits unrestricted use, distribution, and reproduction in any medium, provided the original work is properly credited. 
Neutrophils constitute the central line of the innate immune defense against many bacteria $[7,8]$. Their arsenal is impressive and ranges from killing and phagocytosis to the production and release of antimicrobials and immunoregulatory cytokines [9,2]. Neutrophils play a critical role in the host defense against B. pseudomallei. Although excessive neutrophil recruitment may be detrimental to the host [10], neutrophils are essential for early bacterial containment. Activated neutrophils are rapidly recruited to the lungs upon infection, and neutrophil depletion leads to accelerated mortality in mice $[11,12]$. Not surprisingly, conditions that predispose individuals to melioidosis, most notably diabetes mellitus, are associated with impaired neutrophil function [3,13-15].

Neutrophil extracellular traps (NETs; another effector mechanism of neutrophils) consist of extracellular DNA and histone-based structures decorated by antimicrobials such as neutrophil elastase and myeloperoxidase. They ensnare bacteria, degrade virulence factors, and ultimately kill their target [16]. Besides their antimicrobial function, NETs also induce a strong procoagulant response via extracellular nucleosomes (extracellular frames consisting of DNA and histones) within the NETs, which stimulates the proteolytic activity of neutrophil elastase and in turn promotes coagulation [17-20]. Virtually, all microbes that cause sepsis (including B. pseudomallei) have been shown to induce NET formation in vitro $[7,21,16]$. In a mouse model of intraperitoneal Escherichia coli infection, it was recently shown that NETs released into the vasculature were able to trap bacteria from the bloodstream and prevent bacterial dissemination [22]. Intriguingly, some bacteria (such as certain strains of Streptococcus pneumoniae and Pseudomonas aeruginosa) have developed mechanisms to circumvent NET-mediated killing, for example, via DNase $[21,23,8,19]$. In theory, overwhelming NETosis or a reduced clearance capacity of NETs could be detrimental to the host during sepsis and contribute to ongoing inflammation, organ damage, and/or exhaustion of the immune system [21]. In the context of sepsis, free circulating DNA is regarded as an endogenous danger signal or 'danger-associated molecular pattern' (DAMP). DAMPs are released during inflammatory stress and trigger the host immune response [24-26]. The role and consequences of NET formation during human sepsis, however, remain ill-defined.

In this study, we examined the expression of NET-related markers in patients with melioidosis. We examined next the functional role of NETs in an in vitro model of B. pseudomallei infection, then examined the role of NETs in vivo by treating mice infected with B. pseudomallei with DNase to disrupt the DNA backbone of NETs.

\section{Methods}

\section{Studies of clinical melioidosis}

The clinical study was approved by the Oxford Tropical Research Ethics Committee (OXTREC 018-07) and the Ethics Committee of Mahidol University (MUTM 2008001-01). This co-host has been described previously [27]: 44 patients with culture-proven melioidosis and sepsis, from whom ethylenediaminetetraacetic acid (EDTA) plasma was obtained on day of recruitment, 7 days after, and at a follow-up clinic $\geq 28$ days after discharge. Patients were recruited within $48 \mathrm{~h}$ of admission. All patients had cultureproven melioidosis and had two out of four criteria for systemic inflammatory response syndrome (SIRS) [28]. Eligible patients had received active antimicrobial chemotherapy for less than $48 \mathrm{~h}$ (ceftazidime, amoxicillin-clavulanate, meropenem, or imipenem). 
Thirty-four patients were classed as diabetic if they had an HbA1c $\geq 7.8 \%$ at enrollment or a diagnosis of diabetes made prior to admission. Eighty-two healthy subjects served as controls (52 with diabetes and 30 without; Additional file 1: Table S1).

Peripheral leukocyte gene expression was determined via microarray analysis, as described previously [29]. RNA was assayed using HumanWG-6 v3.0 Expression BeadChips (Illumina ${ }^{\circ}$, Illumina Inc., San Diego, CA, USA), and data have been deposited at ArrayExpress, EMBL-EBI (accession-number E-TABM-852-n). We interrogated this database for proteins co-localizing to NETs [17]. To assess human neutrophil elastase, complexes together with $\alpha 1$-antitrypsin were assayed in plasma by ELISA, as elastase in plasma is immediately inactivated by the formation of these covalent complexes and is hardly detectable in its active form [30,31]. Nucleosome levels were determined in EDTA plasma by enzyme-linked immunosorbent assay (ELISA) [32,33]. In brief, the capture antibody was CLB-ANA/60 (which recognizes histone-3). The detector antibody was biotinylated CLB-ANA/58 (which recognizes an epitope exposed on complexes of histone-2A, histone-2B, and dsDNA). The reaction was read using poly-HRP and $3,3^{\prime}, 5,5^{\prime}$-tetramethylbenzidine as the chromogenic substrate.

\section{Human neutrophil stimulation}

B. pseudomallei strain 1026b [34] was cultured overnight and transferred to fresh Luria broth for $3 \mathrm{~h}$ in a $37^{\circ} \mathrm{C}$ shaker to yield bacteria at mid log-phase. Staphylococcus aureus strain LAC (USA 300; a known NET inducer [35]) was grown to stationary phase for $20 \mathrm{~h}$ in tryptic soy and brain-heart infusion broth at $37^{\circ} \mathrm{C}$. Bacteria were centrifuged for 2 min at $300 \times g$ and the resulting pellet resuspended in Hanks' balanced salt solution (HBSS) with $\mathrm{Ca}^{2+}$ and $\mathrm{Mg}^{2+}$ (HBSS+/+; Gibco, Invitrogen, Carlsbad, CA, USA) before use.

Human neutrophils were isolated from peripheral blood of healthy donors using Polymorphprep $^{\text {tw }}$ (Axis-Shield, Oslo, Norway). Following erythrocyte lysis, neutrophils were washed and resuspended with HBSS without $\mathrm{Ca}^{2+}$ and $\mathrm{Mg}^{2+}$ (HBSS-/-) [7]; $\geq 99 \%$ of neutrophils were viable as determined by trypan blue exclusion [36]. Human NETs were induced as described previously $[7,17]$. In brief, neutrophils were resuspended in HBSS-/- (final concentration $2 \times 10^{5}$ cells $/ 100 \mu \mathrm{L}$ ) and infected with $100 \mu \mathrm{L} \mathrm{HBSS}+/+$ containing live B. pseudomallei (final concentration $2 \times 10^{5}$ to $2 \times 10^{7} \mathrm{cfu}$ ) at $37^{\circ} \mathrm{C}$ and $5 \% \mathrm{CO}_{2}$ to induce NET formation. $\mathrm{HBSS}+/+$ medium alone served as the negative control; $20 \mathrm{nM}$ phorbol 12-myristate 13-acetate (PMA; Sigma-Aldrich Corporation, St. Louis, MO, USA) and $S$. aureus $\left(2 \times 10^{5} \mathrm{cfu}\right)$ served as positive controls. Supernatant was harvested at 0,1 , and $4 \mathrm{~h}$ and passed through a $0.2-\mu \mathrm{m}$ filter (Millipore, Billerica, MA, USA) to remove live bacteria prior to storage at $-20^{\circ} \mathrm{C}$. For DNA quantification, cells were washed once with medium before adding HBSS- $/-, \mathrm{Ca}^{2+}$ and $\mathrm{Mg}^{2+}(5 \mathrm{mM})$, and $0.1 \mathrm{U} / \mathrm{mL}$ DNase (Roche Diagnostics, Indianapolis, IN, USA) and incubated for a further $2 \mathrm{~h}$ at $37^{\circ} \mathrm{C}$. Sterile $20 \mathrm{mM}$ EDTA (Thermo Scientific, Waltham, MA, USA) was added to stop DNase activity. In control wells, EDTA was added prior to incubation with DNase.

To examine the growth-inhibiting properties of NETs on B. pseudomallei, $3 \times 10^{5}$ human neutrophils per well were resuspended in $50 \mu \mathrm{L}$ Iscove's modified Dulbecco's medium (IMDM; Gibco) in a 96-well plate, then stimulated with 100 nM PMA for $5 \mathrm{~h}$ at $37^{\circ} \mathrm{C}\left(5 \% \mathrm{CO}_{2}\right)$ to induce NET formation [7]. Following NET formation, the cells were preincubated for $30 \mathrm{~min}$ at $37^{\circ} \mathrm{C}$ with $50 \mathrm{U}$ DNase, heat-inactivated DNase $\left(80^{\circ} \mathrm{C}\right.$ 
for $10 \mathrm{~min}$ ), or phosphate-buffered saline (PBS). NETs were stained with Sytox Green $5 \mathrm{mM}$ (Molecular Probes, Eugene, OR, USA) and then visualized using a confocal microscope (Olympus IX81, Olympus Corporation, Tokyo, Japan). The supernatant was discarded, and approximately $7 \times 10^{2} \mathrm{cfu} / 100 \mu \mathrm{L}$ IMDM of log-phase B. pseudomallei were added to the wells and spun down for $5 \mathrm{~min}$ at $300 \times \mathrm{g}$. After $10 \mathrm{~h}$, bacterial loads were quantified by culture on horse blood agar.

Free elastase in the supernatant was assayed by ELISA as in the absence of plasma no complexes are formed with $\alpha 1$-antitrypsin, as described previously $[37,30]$. Since NET formation is quantified by the measurement of DNA release after DNase treatment, and DNA will degrade the epitope of the anti-nucleosome antibody, measurement of nucleosomes by this ELISA is not suitable [38]. Therefore extracellular DNA was quantified in the supernatant using a Quant-i $\mathrm{T}^{\mathrm{mm}}$ Picogreen $^{\circ}$ dsDNA Assay Kit (Invitrogen) according to the manufacturer's instructions and was analyzed with a SpectraFluor Plus absorbance reader (Tecan Group, Männedorf, Switzerland).

\section{Mouse experiments}

Mouse experiments were approved by the Academic Medical Center Animal Use and Welfare Committee (DIX16AB). Pathogen-free 8- to 12-week-old C57BL/6 mice (Charles River, Wilmington, MA, USA) were inoculated intranasally with $1,000\left(\mathrm{LD}_{100}\right)$ or $125\left(\mathrm{LD}_{50}\right) \mathrm{cfu}$ B. pseudomallei 1026b in a 50- $\mu \mathrm{L}$ saline solution (eight per group) as described previously $[39,40]$. Mice were treated intravenously with 2,000 U DNase I (Roche) in $200 \mu \mathrm{L}$ PBS at 0, $4,20,26,42$, and $50 \mathrm{~h}$ post-inoculation to disrupt the NET backbone; control mice received $200 \mu \mathrm{L}$ PBS intravenously as described [22]. Lung, broncheoalveolar lavage fluid (BALF), liver, and EDTA blood were collected for bacterial culture and cytokine assays as described previously [41]. Nucleosomes were assayed in plasma and BALF to quantify NET formation.

Neutrophil counts in BALF were determined by FACS (FACSCalibur; Becton Dickson, San Jose, CA, USA) using directly labeled antibodies against Gr-1 (Ly-6G; Gr-1 FITC; BD Pharmingen, BD Biosciences, San Jose, USA). Nucleosome levels were measured by ELISA $[32,33]$. Tumor necrosis factor (TNF)- $\alpha$, interleukin (IL)-6, IL-10, and chemokine (C-X-C motif) ligand 1 (CXCL1 or $\mathrm{KC}$ ) in lung homogenates were measured by sandwich ELISA (R\&D Systems, Minneapolis, MN, USA) [41]. Plasma cytokine concentrations (TNF- $\alpha$, IL-6, interferon (IFN)- $\gamma$, IL-10, monocyte chemotactic protein-1 [MCP-1 or CCL2], and IL-12p70) were measured by cytometric bead array (BD Biosciences). Lactate dehydrogenase (LDH), aspartate aminotransferase (AST), alanine transaminase (ALT), urea, and creatinine were measured in plasma with spectrophotometry (Roche Diagnostics). Lung and liver inflammation were scored histologically as described previously [42,41,40]. In brief, lung inflammation and damage of tissue were analyzed with respect to the following parameters: surface with pneumonia, necrosis and/or formation of abscess, interstitial inflammation, endothelialitis, bronchitis, edema, thrombus formation, and pleuritis. Liver pathology was analyzed with respect to the following parameters: area of liver with parenchymal inflammation, necrosis and/or abscess formation, portal inflammation, and thrombus formation.

\section{Statistical analysis}

Gene expression data were analyzed using R-Bioconductor, as described previously [29]. We log-transformed neutrophil elastase and nucleosome concentrations to correct 
for heteroscedasticity. For correlations, Pearson $r$ is reported. In the kinetic assay, $P$ values reported are for the slope of the linear regression model with elastase or DNA as the dependent variable and time point or stimulus as independent variables. Differences between murine groups were analyzed by the Mann-Whitney $U$ or unpaired $t$ test where appropriate. Analyses were done in GraphPad Prism version 6.0 for Mac OS X (GraphPad Software), and data are expressed as mean and SD unless stated otherwise.

\section{Results}

Nucleosome and neutrophil elastase levels are markedly elevated in patients with melioidosis and correlate with disease severity

To establish the presence of NETs during clinical melioidosis, we measured nucleosomes (complexes of extracellular DNA and histones and a useful measure of cell-free DNA in plasma) and neutrophil elastase (a measure of neutrophil degranulation) in the plasma of 44 patients with septic melioidosis and 82 healthy controls. Both are key NET-related markers $[17,18,43]$, and both nucleosome $(P<0.0001)$ and elastase levels $(P<0.0001)$ were abundant in the plasma of patients with melioidosis on day of recruitment compared to healthy controls (Figure 1A,D).

The mortality rate of $27 \%$ ( 12 of 44$)$ in this cohort enabled us to correlate the presence of these NET-related markers with disease severity. No differences were seen in the admission nucleosome and neutrophil elastase levels when comparing survivors and non-survivors (Figure 1B,E). No differences were seen in concentration levels of both NET markers in non-survivors on day of enrollment and day 7. All non-survivors died before the 28 day time point. However, a marked difference in nucleosome

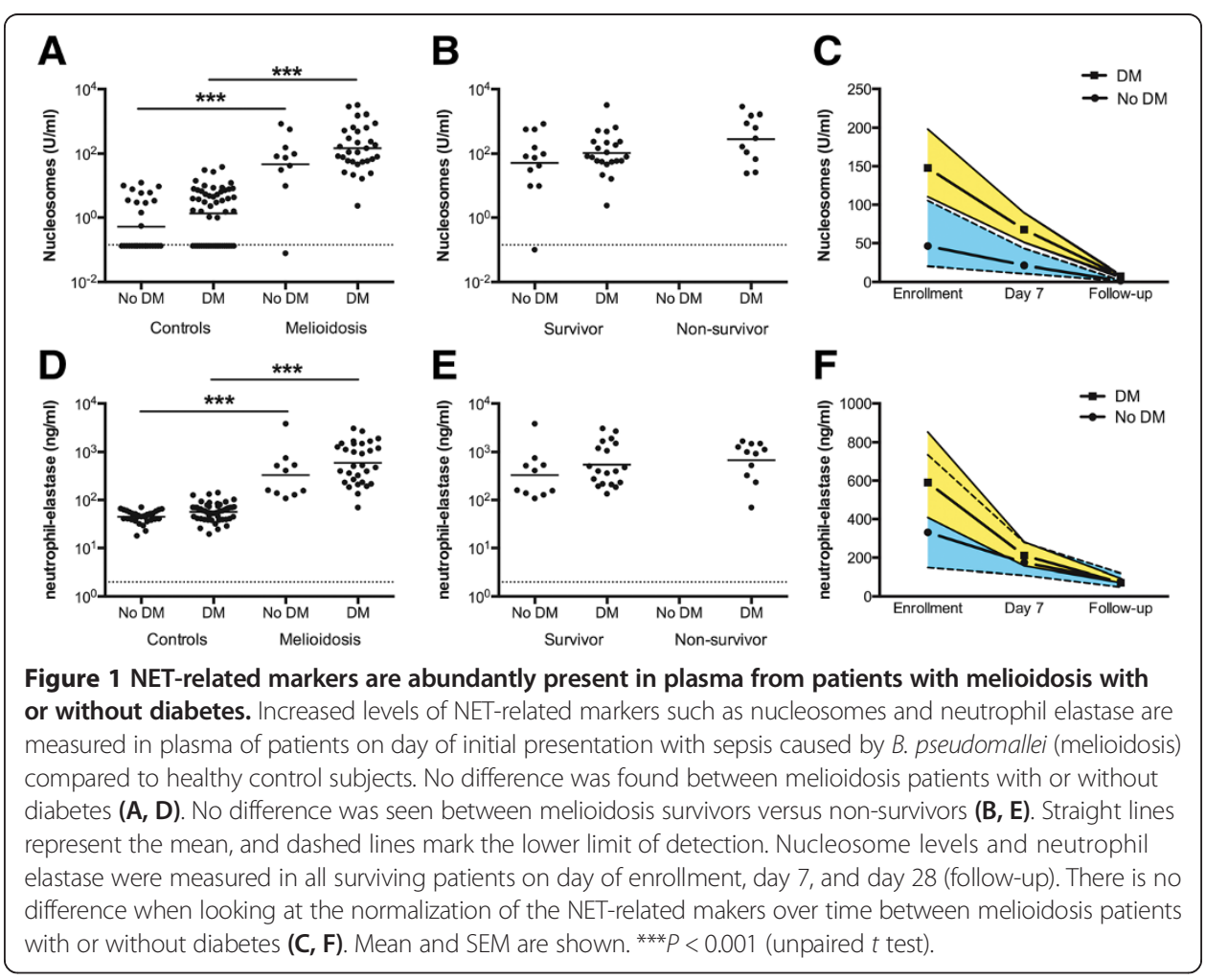


concentrations between survivors and non-survivors at day 7 was seen: at this time point, patients that went on to die had significantly higher nucleosome levels $(192.3 \pm$ 5) compared to survivors $(33.6 \pm 4 ; P=0.001)$. Evidence for an association between nucleosome and neutrophil elastase plasma levels and disease severity was obtained in patients who survived, a second blood sample was drawn during treatment (at day 7) and after successful completion of therapy ( $\geq 28$ days from discharge). In all surviving patients, plasma nucleosome and elastase concentrations moved toward normal (Figure 1C,F). Moreover, neutrophil activation (evidenced by elastase- $\alpha_{1}$-antitrypsin complexes) was strongly correlated with nucleosome levels at admission $(P<0.001$, Figure 2$)$, supporting the hypothesis that neutrophils are the source of nucleosomes. Of note, gene expression of proteins known to be associated with NET formation [17] was determined in total peripheral leukocytes derived from patients with culture-confirmed septic melioidosis and healthy controls. In line with the protein measurements, a marked up-regulation of genes encoding the central NET-associated proteins nucleosome histone-2B and histone-4 was found (Additional file 2: Table S2).

\section{No influence of diabetes on the release of the NET-related markers}

Since diabetes is the main risk factor for melioidosis and diabetes is known to negatively influence neutrophil function $[3,15,14]$, we next sought to determine whether the presence of diabetes influenced the release of NET-related markers in our cohort of patients of whom $72 \%$ were diabetic. We found no difference in the release of the NET-related markers in melioidosis when comparing patients with or without diabetes on day of recruitment. In addition, NET-related markers were not different in healthy controls with or without diabetes (Figure 1A,D).

Elastase and extracellular DNA are released by neutrophils in a dose- and time-dependent fashion upon stimulation with $B$. pseudomallei

To examine whether neutrophils were the common source of NET-related marker release in patients with melioidosis, we isolated human neutrophils from healthy individuals and stimulated them with various known NET inducers as well as B. pseudomallei upon which

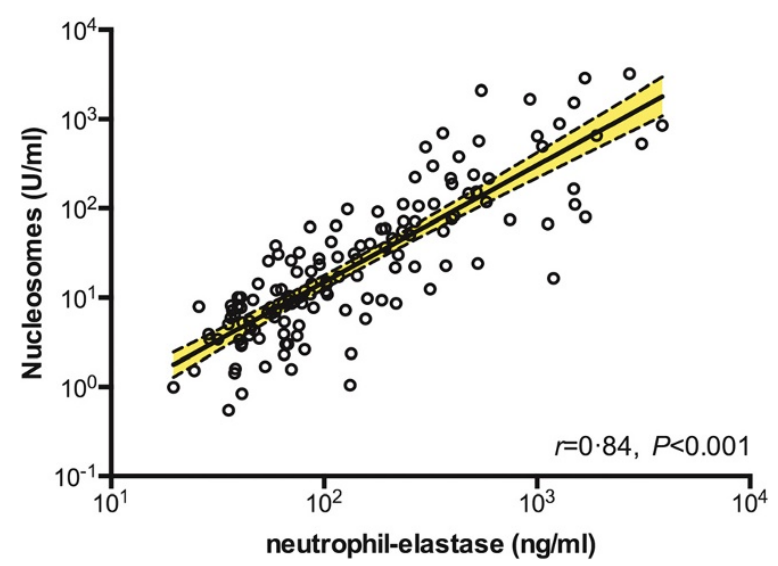

Figure $\mathbf{2}$ Neutrophil elastase and nucleosome release are highly correlated in patients with melioidosis. Linear regression of neutrophil elastase versus nucleosomes. Correlation coefficient reported is for Pearson's $r$. 
the kinetics of elastase and extracellular DNA in culture supernatants was determined. Consistent with the patient data, elastase levels increased following stimulation with B. pseudomallei, and this occurred in a dose-dependent $(P<0.001$, Figure $3 \mathrm{~A})$ and timedependent manner $(P=0.005$, Figure $3 C)$. Extracellular DNA release also increased in a time- and dose-dependent manner following B. pseudomallei infection: extracellular DNA concentrations increased as the multiplicity of infection (MOI) increased $(P<0.001$, Figure 3C) and over time $(P<0.001$, Figure 3D). Of note, B. pseudomallei-infected cells released similar amounts of elastase and DNA as $S$. aureus (Additional file 3: Figure S1), itself a potent NET inducer [44]. Elastase levels and DNA release were also strongly correlated, reflecting the clinical data (Figure 3E).

\section{NETs exhibit antibacterial activity against $B$. pseudomallei}

We next sought to examine whether activated NETs were able to kill B. pseudomallei. We pre-stimulated human neutrophils with PMA for $5 \mathrm{~h}$ to induce NET formation $[7,17]$, then preincubated the cells with PBS or DNase prior to infection with $B$. pseudomallei. Extracellular degradation of DNA by DNase treatment was confirmed by confocal microscopy (Figure 4A,B). Disrupting the backbone of the NETs with active DNase impaired the killing of B. pseudomallei by NETs from human neutrophils (Figure 4C). Taken together, these data confirm the involvement of NETs in the killing of B. pseudomallei by neutrophils.

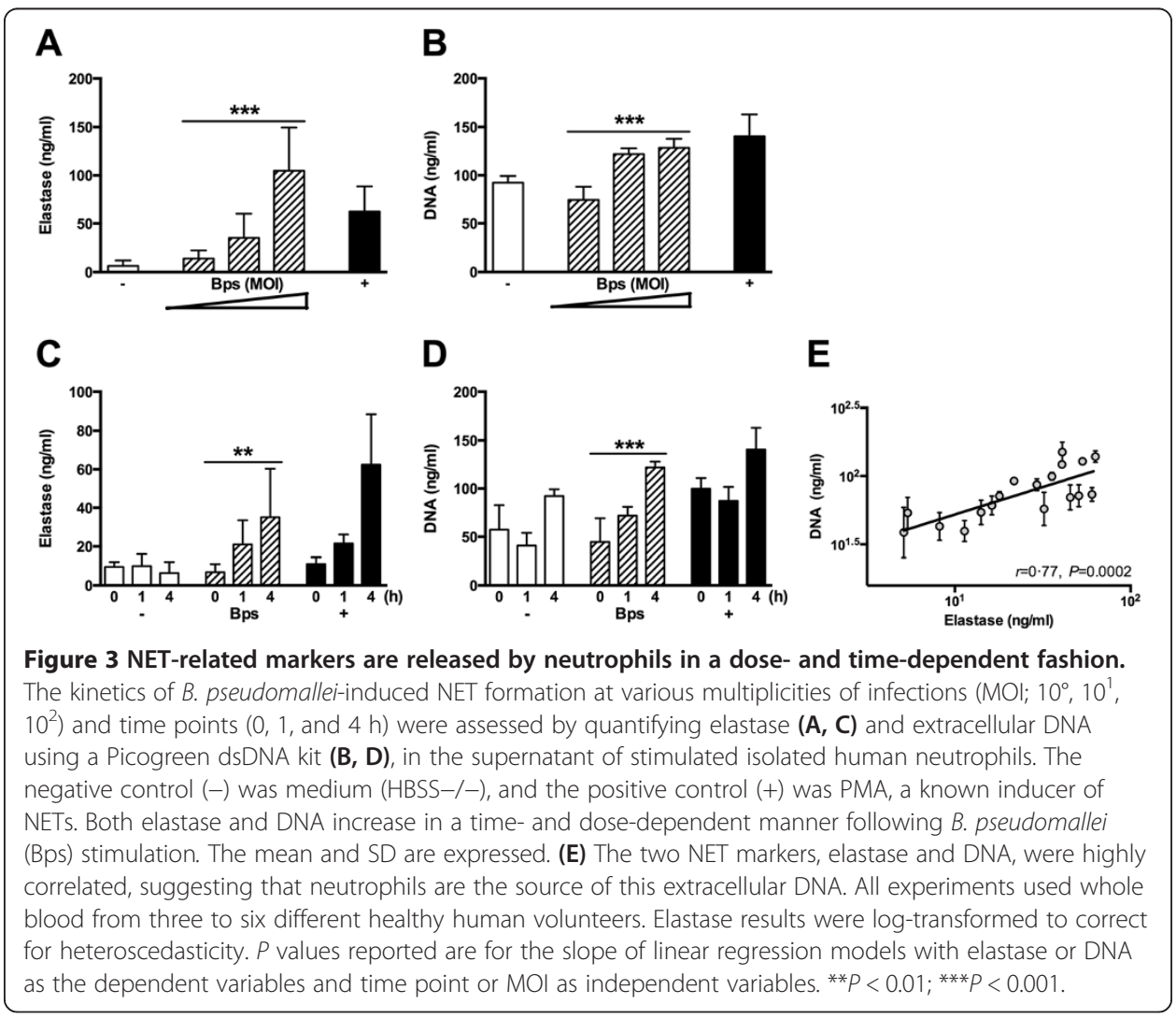



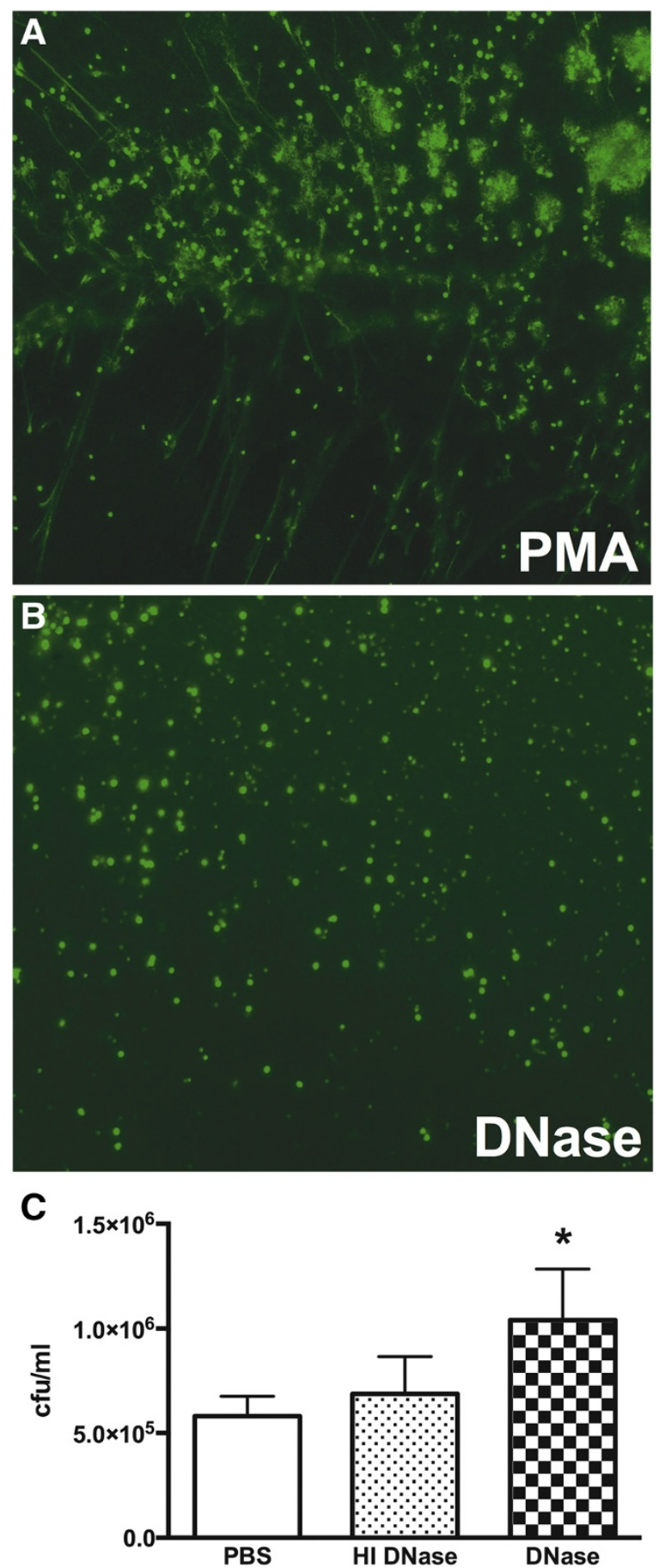

Figure 4 NETs exhibit antibacterial activity against B. pseudomallei. Representative confocal micrographs of human neutrophils stimulated with PMA (A) to produce NETs and post-stimulation treated with PBS, heat-inactivated (HI) DNase, or active DNase (B), before infection with $7 \times 10^{2} \mathrm{cfu}$ B. pseudomallei (C). Data are expressed as mean and SD. $P$ value was determined via one-way ANOVA. Blood from three different healthy human volunteers was used.

NETs do not protect against bacterial dissemination in B. pseudomallei-induced sepsis Having confirmed that NETs are capable of killing of B. pseudomallei in vitro, we sought to replicate this finding in a murine model of melioidosis. We infected mice 
with a lethal challenge of $B$. pseudomallei $\left(10^{3} \mathrm{cfu}\right)$ and treated half of them with intravenous DNase twice daily in order to disrupt NET formation, as described previously [22]. Animals were sacrificed after 24 or $72 \mathrm{~h}$ to determine bacterial loads in BALF and lung (primary sites of the infection), and the liver and blood (to evaluate the extent of bacterial dissemination). In this murine model of Gram-negative sepsis, untreated mice showed an increase of nucleosome levels in plasma (17.6 \pm 15$)$ and BALF $(202.7 \pm 110)$ $24 \mathrm{~h}$ post-infection (Figure 5A,D). In comparison, uninfected untreated mice $(t=0)$ had low to undetected plasma and BALF nucleosome levels (5.6 \pm 3 and $12.9 \pm 5$, respectively). DNase treatment resulted in successful disruption of intravascular NET formation, as illustrated by significantly decreased nucleosome levels in both plasma (5.3 $\pm 3, P=0.03)$ and BALF $(93.9 \pm 29, P=0.02)$ compared to untreated mice $24 \mathrm{~h}$ post-infection (Figure 5A,D). Disruption of intravascular NETs did not, however, result in changes in bacterial loads in BALF, lung, blood, or liver (Figure 5). In line with the bacterial loads, no differences were seen in total cell count or neutrophil influx in BALF between DNase-treated and untreated mice after infection (data not shown). Because cytokines are important regulators of the inflammatory response to bacterial pneumonia [45], we measured pulmonary (TNF- $\alpha$, IL-6, IL-10, CXCL1) and systemic (TNF- $\alpha$, MCP-1, IL-6, IFN- $\gamma$, IL-10, IL12p70) cytokine and chemokine levels (Table 1). Levels of all cytokines were elevated in both pulmonary and systemic compartments (with the exception of IL-10) upon B. pseudomallei infection. However, in complete correspondence with bacterial loads and cellular influx, no differences were seen between the DNase-treated animals and controls.

We speculated that the infectious challenge might have been too high to reveal an effect of NET blockade in our model and therefore repeated the experiments with a lower inoculum $\left(1.25 \times 10^{2} \mathrm{cfu}\right.$ which is the $\left.\mathrm{LD}_{50}\right)$. Again, although significant reductions

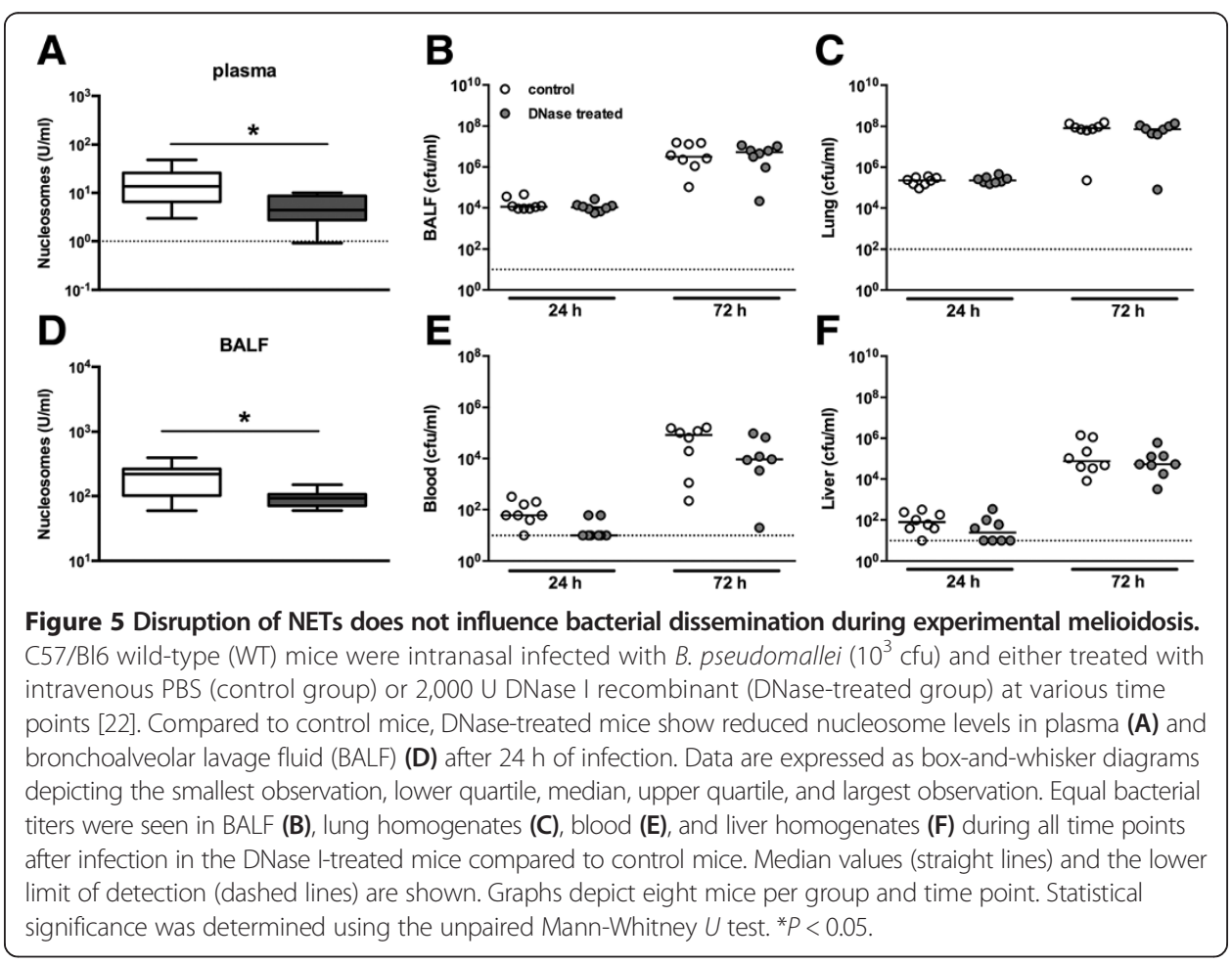


Table 1 Cytokines and markers for systemic organ injury do not differ between mice treated with intravenous DNase or PBS post-infection with B. pseudomallei

\begin{tabular}{|c|c|c|c|c|c|c|}
\hline & \multicolumn{3}{|c|}{$24 \mathrm{~h}$} & \multicolumn{3}{|c|}{$72 \mathrm{~h}$} \\
\hline & Control & DNase & $P$ value & Control & DNase & $P$ value \\
\hline \multicolumn{7}{|l|}{ Plasma } \\
\hline TNF-a (pg/mL) & $3.3 \pm 2$ & $4.1 \pm 2$ & 0.41 & $407 \pm 319$ & $328.3 \pm 300$ & 0.62 \\
\hline MCP-1 (pg/mL) & $11.7 \pm 13$ & $21.5 \pm 8$ & 0.09 & $3,976 \pm 2,183$ & $2,879 \pm 1,937$ & 0.31 \\
\hline IL-12p70 (pg/mL) & ND & ND & & $8.6 \pm 10$ & $21.6 \pm 20$ & 0.13 \\
\hline IL-6 (pg/mL) & $115.3 \pm 73$ & $104 \pm 62$ & 0.74 & $8,022 \pm 3,814$ & $6,990 \pm 4,252$ & 0.62 \\
\hline $\mathrm{IFN}-\gamma(\mathrm{pg} / \mathrm{mL})$ & $4.0 \pm 6$ & $4.8 \pm 4$ & 0.76 & $1,914 \pm 1,152$ & $1,791 \pm 1,490$ & 0.86 \\
\hline IL-10 (pg/mL) & ND & ND & & ND & ND & \\
\hline AST $(U / L)$ & $63.6 \pm 6$ & $74.4 \pm 37$ & 0.83 & $356.6 \pm 191$ & $247 \pm 143$ & 0.50 \\
\hline $\operatorname{ALT}(U / L)$ & $21.7 \pm 9$ & $27 \pm 24$ & 0.92 & $155.4 \pm 59$ & $138 \pm 98$ & 0.60 \\
\hline $\mathrm{LDH}(\mathrm{U} / \mathrm{L})$ & $210 \pm 74$ & $312.9 \pm 157$ & 0.21 & $1,115 \pm 758$ & $873.2 \pm 375$ & 0.71 \\
\hline Urea (mmol/L) & $9.8 \pm 2$ & $7.7 \pm 2$ & 0.04 & $15.9 \pm 10$ & $14.4 \pm 5$ & 0.91 \\
\hline Creatinine $(\mu \mathrm{mol} / \mathrm{L})$ & $11.3 \pm 2$ & $9.6 \pm 4$ & 0.43 & $15.7 \pm 17$ & $8.4 \pm 7$ & 0.53 \\
\hline \multicolumn{7}{|l|}{ Lung } \\
\hline TNF-a (pg/mL) & $239.1 \pm 110$ & $427.8 \pm 100$ & 0.003 & $3,182 \pm 1,440$ & $3,261 \pm 1,382$ & 0.91 \\
\hline IL-6 (pg/mL) & $945.9 \pm 348$ & $1,103 \pm 201$ & 0.29 & $8,601 \pm 3,329$ & $7,521 \pm 3,482$ & 0.54 \\
\hline $\mathrm{IL}-10(\mathrm{pg} / \mathrm{mL})$ & $205.8 \pm 147$ & $266.4 \pm 57$ & 0.29 & $559.4 \pm 219$ & $498.3 \pm 107$ & 0.49 \\
\hline CXCL1 $(\mathrm{pg} / \mathrm{mL})$ & $6,771 \pm 4,247$ & $6,147 \pm 2,284$ & 0.72 & $26,421 \pm 10,123$ & $26,305 \pm 10,450$ & $>0.99$ \\
\hline
\end{tabular}

Cytokines and markers for systemic organ injury were measured in plasma and/or lung homogenates 24 and $72 \mathrm{~h}$ post-infection. Data are means \pm SD (cytokines) or median (range) of five to eight mice per group. Actual $P$ values are shown, determined via $t$ test or Mann-Whitney $U$ where appropriate; ND, not detectable or below detection limit; TNF- $a$, tumor necrosis factor-alpha; MCP-1, monocyte chemo-attractive protein-1; IL, interleukin; IFN-y, interferon-gamma; AST, aspartate transaminase; ALT, alanine transaminase; $\mathrm{LDH}$, lactate dehydrogenase; $\mathrm{CXCL1}$, chemokine (C-X-C motif) ligand 1.

of nucleosomes of DNase-treated mice were seen in BALF $(P=0.002)$ compared to untreated mice, no differences were seen in bacterial burdens in BALF, lung, blood, or liver nor local or systemic inflammatory cytokine levels $72 \mathrm{~h}$ post-infection (data not shown). These results indicate that disrupting NET release by degradation of the DNA backbone does not impair the host defense against B. pseudomallei infection.

Intravascular NETs cause collateral liver damage during experimental melioidosis

It has been reported previously that NETs are implicated in tissue damage during $E$. coli sepsis [22]. We therefore examined lung and liver tissue histologically for inflammation. No differences in lung pathology scores were observed between groups (Figure 6A,B,C). In accordance with a previous report, however, we found that significantly less liver inflammation was seen in the DNase-treated group when compared to controls at $72 \mathrm{~h}$ post-infection $(P=0.04$, Figure $6 \mathrm{D}, \mathrm{E}, \mathrm{F})$. It should be noted, however, that serum levels of AST and ALT, both markers of hepatocellular injury, as well as urea, creatinine, and LDH, markers of kidney and systemic organ injury, showed a trend toward lower values in the DNase-treated mice, although this did not reach statistical significance (Table 1). We conclude that there is only a limited role for NETs during B. pseudomallei infection in vivo.

\section{Discussion}

NETs have emerged as key players in the innate host response to many bacteria. Neutrophils release extracellular DNA (including nucleosomes) and neutrophil elastase to 


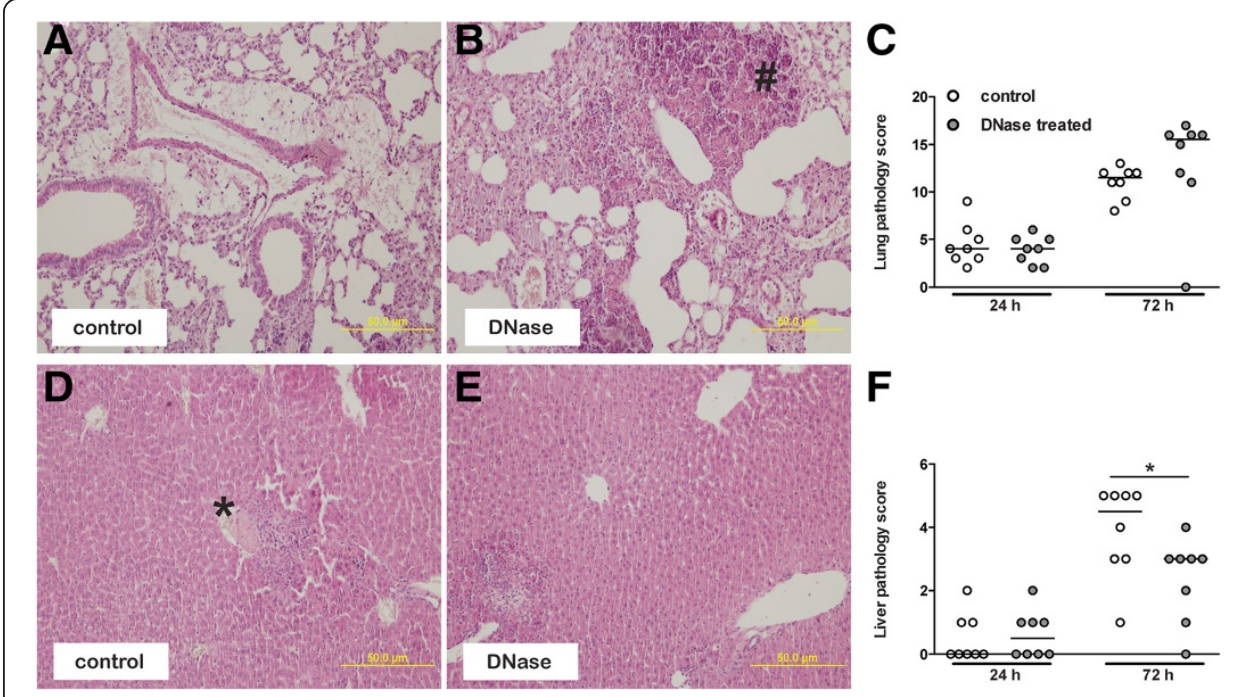

Figure 6 Effect of DNase treatment on pulmonary and hepatic inflammation after infection with $B$. pseudomallei. Representative hematoxylin and eosin stained slides of lung (A, B) and liver (D, E) of control and DNase-treated mice $72 \mathrm{~h}$ post-infection with B. pseudomallei $\left(10^{3} \mathrm{cfu}\right.$ ). Marked inflammation was seen post-infection, and areas of necrosis (marked with a number sign) and thrombosis formation (marked with an asterisk) were seen in both groups. Significantly less liver inflammation was observed in the DNase-treated group when compared to controls $72 \mathrm{~h}$ after infection. Total organ pathology score (lung and liver (C, F)) was determined 24 and $72 \mathrm{~h}$ post-infection according to the scoring system as described $[41,39,40]$. Graphs depict eight mice per group and time point. Median values are shown, and statistical significance was determined using the unpaired Mann-Whitney $U$ test. ${ }^{*} P<0.05$.

entrap and capture bacteria [16], and animals depleted of neutrophils are markedly more susceptible to B. pseudomallei infection [12]. Our study extends the description of the role of NETs in the host response to Gram-negative sepsis caused by B. pseudomallei in both clinical melioidosis and a murine model of melioidosis. We found that the key NET components, nucleosomes and neutrophil elastase, were both elevated in clinical melioidosis. In vitro observations supported the notion that neutrophils are the source of these proteins and isolated human neutrophils infected with B. pseudomallei released NET-related markers in a time- and dose-dependent manner. We further showed that NETs killed B. pseudomallei in an in vitro human neutrophil infection model, although in a murine model of experimental melioidosis, in which DNase was used to disrupt NET formation, no protective role for NETs in the host defense against B. pseudomallei infection could be demonstrated.

Our data corroborate a previous report in which it was shown that bactericidal NETs were released from human neutrophils in response to B. pseudomallei in an in vitro model [46]. In addition, we recently showed that inhibition of endogenous activated protein $\mathrm{C}$, which exerts cytoprotective effects on the endothelium by cleavage of histones [47], leads to excessive nucleosome release in BALF during murine experimental melioidosis [48]. We now demonstrate that there is an abundant release of NET-related markers in plasma from melioidosis patients. In vitro experiments demonstrated that NET-related markers are released by neutrophils and exhibit NET-dependent antibacterial activity upon infection with B. pseudomallei.

Surprisingly, we found that NETs played no role in bacterial clearance in our experimental mouse model of sepsis. In DNase-treated mice, circulating nucleosomes were decreased but 
there was no difference in cellular influx nor bacterial counts and cytokine release. This also argues against a role for nucleosomes (which can become harmful to the host when released in excessive amounts $[25,26])$ as significant endogenous danger signals in melioidosis. This correlates with our clinical finding that the concentration of circulating NET markers in melioidosis patients was not different between survivors and non-survivors on day of admission. We did however find a difference between survivors and non-survivors on day 7 , which is in line with our previous finding that circulating nucleosomes correlated with severity of the inflammatory response and outcome in a cohort of children suffering from meningococcal sepsis [49].

ELISAs detecting nucleosomes have proven to be a good indicator of NET release in patients [43] but are not specific for nucleosomes released by neutrophils. We therefore cannot exclude that nucleosomes released into the circulation by other cell types, such as endothelial and parenchymal cells, are detected as well. However, the positive correlation with neutrophil elastase together with the marked up-regulation of genes encoding the central NET-associated proteins does suggest that neutrophils are the main source of the detected nucleosomes. Although B. pseudomallei can induce NET release, NETs are predominantly a mechanism for clearing extracellular pathogens, and $B$. pseudomallei may escape NETosis by hiding intracellularly [50,21,51]. Indeed, it was found that both the type 3 secretion system (an important virulence factor of $B$. pseudomallei) and the bacterial capsule may play a role in evading NETs [46]. Furthermore, one could hypothesize that like S. aureus [52], B. pseudomallei is able to cause leukocyte toxicity by converting NETs into a bioproduct thereby inducing macrophage apoptosis. However, this mechanism would be unfavorable for B. pseudomallei as it is already known that macrophage destruction is one of the host immune defense strategies to restrict intracellular growth of B. pseudomallei [2]. Another possibility could be that plasma from patients with severe sepsis induces plateletneutrophil interactions in a TLR4-dependent fashion leading to the production of NETs $[53,22]$, whereas TLR4 does not seem to play an important role in the clearance of B. pseudomallei $[2,41]$.

At least a third of all patients with melioidosis have diabetes mellitus as a predisposing factor $[3,1,2]$. An explanation for this increased susceptibility was sought in the function of neutrophils from diabetic patients, which displayed impaired phagocytosis of $B$. pseudomallei, reduced migration, and the inability to produce an oxidative burst to kill intracellular bacteria [54]. Furthermore, it was shown that isolated neutrophils from patients with diabetes mellitus released less extracellular DNA upon in vitro stimulation with B. pseudomallei, which was associated with a reduced bacterial killing capacity when compared to neutrophils derived from healthy controls [46]. However, in our study, we did not observe a difference in the release of NET-related markers between diabetic and non-diabetic melioidosis patients. The combination of clinical data and data from our murine model argues against an important role for the NETs in melioidosis. Our data also suggest that impaired NET release does not provide an explanation for the increased susceptibility to B. pseudomallei infection in patients with diabetes.

NET formation may also injure the host [21]: our finding that DNase-treated mice have diminished hepatocellular injury after infection is consistent with previous reports in which mice deficient in NET components have decreased hepatocellular inflammation compared to controls in an E. coli-induced sepsis model [55,22]. 


\section{Conclusion}

We have shown that B. pseudomallei is a potent inducer of NETosis, as reflected by increased concentrations of NET-related components in patients with melioidosis. Although NETs exhibit antibacterial activity against B. pseudomallei in vitro, NET formation does not protect against bacterial dissemination and inflammation in a murine model of B. pseudomallei infection. This correlates with our clinical finding that levels of NET components do not correlate with mortality.

\section{Additional files}

Additional file 1: Table S1. Characteristics of study subjects.

Additional file 2: Table S2. Proteins that localize to NETs in peripheral leucocytes from patients with melioidosis.

Additional file 3: Figure S1. B. pseudomallei is as potent as S. aureus in the induction of NETosis. Isolated human neutrophils were stimulated with either Burkholderia pseudomallei (Bps) or Staphylococcus aureus (SA) for $4 \mathrm{~h}$ after which elastase (A) and extracellular DNA (B) release were measured. For both bacterial strains, equal $\mathrm{MOls}\left(10^{1}\right)$ were used. Medium (HBSS-/-) served as negative control and PMA, a known inducer of NETs, as positive control. Extracellular DNA was measured using a Picogreen dsDNA kit. Mean and SDs are shown. P value comparing Bps, $\mathrm{SA}$, and PMA to medium control was determined via unpaired $t$ test after log transformation of the data. Blood from three to six different healthy human volunteers were used. $P$ values ${ }^{*}<0.05,{ }^{* *}<0.01,{ }^{* * *}<0.001$ are shown.

\section{Abbreviations}

ALT: alanine transaminase; AST: aspartate aminotransferase; B. pseudomallei: Burkholderia pseudomallei; BALF: bronchoalveolar lavage fluid; CXCL1/KC: chemokine (C-X-C motif) ligand 1; DAMPS: danger-associated molecular patterns; IFN- $\gamma$ : interferon-gamma; IL: interleukin; LDH: lactate dehydrogenase; MCP-1/CCL2: monocyte chemotactic protein-1; MOI: multiplicity of infection; NET: neutrophil extracellular traps; PMA: phorbol 12-myristate 13-acetate; S. aureus: Staphylococcus aureus; TNF-a: tumor necrosis factor-alpha.

\section{Competing interests}

HKdJ received unrestricted funding for this project from the Academic Medical Center (AMC PhD Scholarship), GCK from Wellcome Trust of Great Britain (086532/Z/08/Z), and WJW from The Netherlands Organisation for Scientific Research (NWO; VENI grant number 91610008) and The Netherlands Organisation for Health Research and Development (ZonMw; Clinical Fellowship grant number 90700424). The other authors have no competing interests.

\section{Authors' contributions}

HKJ, GCK, AA, IB, NPD, SJP, SZ, and WJW conceived and designed the experiments. HKJ, GCK, AA, and AJM performed the experiments. HKJ, GCK, AA, AJM, IB, FS, JJR, SZ, and WJW analyzed the data. NPD, SJP, and SZ contributed reagents/materials/analysis tools. HKJ, GCK, and WJW wrote the first draft. HKJ, GCK, AA, AJM, IB, FS, JJR, NPD, SJP, SZ, and WJW contributed to the writing of the manuscript and agreed with the final manuscript results and conclusions. GCK enrolled patients. All authors read and approved the final manuscript.

\section{Meetings at which work has been presented}

This work was presented in part at the International Federation of Shock Societies meeting, June 2012, Miami Beach, Florida, USA; the 15th International Congress of Immunology, August 2013, Milan, Italy; and the 7th World Melioidosis Congress, September 2013, Bangkok, Thailand.

\section{Acknowledgements}

We are grateful to the valuable contributions of past and present group members for their work contributing to the understanding of neutrophil extracellular traps and bacteria. Furthermore, we would like to thank Tom van der Poll for critically reading the manuscript. We would also like to thank Gerard van Mierlo, Shabnam Solati, Marieke van den Brink, and Joost Daalhuisen for their expert technical assistance.

\footnotetext{
Author details

${ }^{1}$ Center for Experimental and Molecular Medicine (CEMM), Academic Medical Center, Meibergdreef 9, Room G2-132, Amsterdam 1105 AZ, The Netherlands. '2Department of Medicine, Division of infectious diseases, Academic Medical Center, Meibergdreef 9, Room G2-132, Amsterdam 1105 AZ, The Netherlands. 'Department of Medicine, Addenbrooke's Hospital, University of Cambridge, Cambridge CB2 OQQ, UK. ${ }^{4}$ Mahidol-Oxford Tropical Medicine Research Unit, Faculty of Tropical Medicine, Mahidol University, Bangkok 10400, Thailand. ${ }^{5}$ Department of Infection and Tropical Medicine, Heartlands Hospital, Birmingham B9 5SS, UK. ${ }^{6}$ Department of Immunopathology, Sanquin Research, Amsterdam 1066 CX, The Netherlands. ${ }^{7}$ Department of Pathology, Academic Medical Centre, Amsterdam 1105 AZ, The Netherlands. ${ }^{8}$ Nuffield Department of Clinical Medicine, Churchill Hospital, University of Oxford, Oxford OX3 7LJ, UK. ${ }^{9}$ Department of Haematology, Academic Medical Centre, Amsterdam 1105 AZ, The Netherlands.
} 


\section{References}

1. Cheng AC, Currie BJ (2005) Melioidosis: epidemiology, pathophysiology, and management. Clin Microbiol Rev 18(2):383-416

2. Wiersinga WJ, Currie BJ, Peacock SJ (2012) Melioidosis. N Engl J Med 367(11):1035-1044

3. Koh GC, Maude RR, Schreiber MF, Limmathurotsakul D, Wiersinga WJ, Wuthiekanun V, Lee SJ, Mahavanakul W, Chaowagul W, Chierakul W, White NJ, van der Poll T, Day NP, Dougan G, Peacock SJ (2011) Glyburide is anti-inflammatory and associated with reduced mortality in melioidosis. Clin Infect Dis 52(6):717-725

4. Department of Health and Human Services (2012) Possession, use, and transfer of select agents and toxins: biennial review. Federal Register 77:61085-61115

5. Simpson AJ (2001) Melioidosis: a clinical model for gram-negative sepsis. J Med Microbiol 50(8):657-658

6. Wiersinga WJ (2011) Current insights in sepsis: from pathogenesis to new treatment targets. Curr Opin Crit Care 17(5):480-486

7. Achouiti A, Vogl T, Urban CF, Rohm M, Hommes TJ, van Zoelen MA, Florquin S, Roth J, van 't Veer C, de Vos AF, van der Poll T (2012) Myeloid-related protein-14 contributes to protective immunity in gram-negative pneumonia derived sepsis. PLoS Pathog 8(10):e1002987

8. Young RL, Malcolm KC, Kret JE, Caceres SM, Poch KR, Nichols DP, Taylor-Cousar JL, Saavedra MT, Randell SH, Vasil ML, Burns JL, Moskowitz SM, Nick JA (2011) Neutrophil extracellular trap (NET)-mediated killing of Pseudomonas aeruginosa: evidence of acquired resistance within the CF airway, independent of CFTR. PLoS One 6(9):e23637

9. Kolaczkowska E, Kubes P (2013) Neutrophil recruitment and function in health and inflammation. Nat Rev Immunol 13(3):159-175

10. Ceballos-Olvera I, Sahoo M, Miller MA, Del Barrio L, Re F (2011) Inflammasome-dependent pyroptosis and IL-18 protect against Burkholderia pseudomallei lung infection while IL-1 beta is deleterious. PLoS Pathog 7(12):e1002452

11. Wiersinga WJ, Veer C, Wieland CW, Gibot S, Hooibrink B, Day NP, Peacock SJ, van der Poll T (2007) Expression profile and function of triggering receptor expressed on myeloid cells-1 during melioidosis. J Infect Dis 196(11):1707-1716

12. Easton A, Haque A, Chu K, Lukaszewski R, Bancroft GJ (2007) A critical role for neutrophils in resistance to experimental infection with Burkholderia pseudomallei. J Infect Dis 195(1):99-107

13. Suputtamongkol Y, Chaowagul W, Chetchotisakd P, Lertpatanasuwun N, Intaranongpai S, Ruchutrakool T, Budhsarawong D, Mootsikapun P, Wuthiekanun V, Teerawatasook N, Lulitanond A (1999) Risk factors for melioidosis and bacteremic melioidosis. Clin Infect Dis 29(2):408-413

14. Schuetz P, Castro P, Shapiro NI (2011) Diabetes and sepsis: preclinical findings and clinical relevance. Diabetes Care 34(3):771-778

15. Koh GC, Peacock SJ, van der Poll T, Wiersinga WJ (2012) The impact of diabetes on the pathogenesis of sepsis. Eur J Clin Microbiol Infect Dis 31(4):379-388

16. Brinkmann V, Reichard U, Goosmann C, Fauler B, Uhlemann Y, Weiss DS, Weinrauch Y, Zychlinsky A (2004) Neutrophil extracellular traps kill bacteria. Science 303(5663):1532-1535

17. Urban CF, Ermert D, Schmid M, Abu-Abed U, Goosmann C, Nacken W, Brinkmann V, Jungblut PR, Zychlinsky A (2009) Neutrophil extracellular traps contain calprotectin, a cytosolic protein complex involved in host defense against Candida albicans. PLoS Pathog 5(10):e1000639

18. Papayannopoulos V, Metzler KD, Hakkim A, Zychlinsky A (2010) Neutrophil elastase and myeloperoxidase regulate the formation of neutrophil extracellular traps. J Cell Biol 191(3):677-691

19. Engelmann B, Massberg S (2013) Thrombosis as an intravascular effector of innate immunity. Nat Rev Immunol 13(1):34-45

20. Massberg S, Grahl L, von Bruehl ML, Manukyan D, Pfeiler S, Goosmann C, Brinkmann V, Lorenz M, Bidzhekov K, Khandagale AB, Konrad I, Kennerknecht E, Reges K, Holdenrieder S, Braun S, Reinhardt C, Spannagl M, Preissner KT Engelmann B (2010) Reciprocal coupling of coagulation and innate immunity via neutrophil serine proteases. Nat Med 16(8):887-896

21. Kaplan MJ, Radic M (2012) Neutrophil extracellular traps: double-edged swords of innate immunity. J Immunol 189(6):2689-2695

22. McDonald B, Urrutia R, Yipp BG, Jenne CN, Kubes P (2012) Intravascular neutrophil extracellular traps capture bacteria from the bloodstream during sepsis. Cell Host Microbe 12(3):324-333

23. Beiter K, Wartha F, Albiger B, Normark S, Zychlinsky A, Henriques-Normark B (2006) An endonuclease allows Streptococcus pneumoniae to escape from neutrophil extracellular traps. Curr Biol 16(4):401-407

24. Chan JK, Roth J, Oppenheim JJ, Tracey KJ, Vogl T, Feldmann M, Horwood N, Nanchahal J (2012) Alarmins: awaiting a clinical response. J Clin Invest 122(8):2711-2719

25. Chen GY, Nunez G (2010) Sterile inflammation: sensing and reacting to damage. Nat Rev Immunol 10(12):826-837

26. Pisetsky DS (2012) The origin and properties of extracellular DNA: from PAMP to DAMP. Clin Immunol 144(1):32-40

27. Koh GC, Meijers JC, Maude RR, Limmathurotsakul D, Day NP, Peacock SJ, van der Poll T, Wiersinga WJ (2011) Diabetes does not influence activation of coagulation, fibrinolysis or anticoagulant pathways in Gram-negative sepsis (melioidosis). Thromb Haemost 106(6):1139-1148

28. Levy MM, Fink MP, Marshall JC, Abraham E, Angus D, Cook D, Cohen J, Opal SM, Vincent JL, Ramsay G (2003) 2001 SCCM/ESICM/ACCP/ATS/SIS International Sepsis Definitions Conference. Crit Care Med 31(4):1250-1256

29. Koh GC, Schreiber MF, Bautista R, Maude RR, Dunachie S, Limmathurotsakul D, Day NP, Dougan G, Peacock SJ (2013) Host responses to melioidosis and tuberculosis are both dominated by interferon-mediated signaling. PLoS One 8(1):e54961

30. Zeerleder S, Caliezi C, van Mierlo G, Eerenberg-Belmer A, Sulzer I, Hack CE, Wuillemin WA (2003) Administration of C1 inhibitor reduces neutrophil activation in patients with sepsis. Clin Diagn Lab Immunol 10(4):529-535

31. van Montfoort ML, Stephan F, Lauw MN, Hutten BA, Van Mierlo GJ, Solati S, Middeldorp S, Meijers JC, Zeerleder S (2013) Circulating nucleosomes and neutrophil activation as risk factors for deep vein thrombosis. Arterioscler Thromb Vasc Biol 33(1):147-151

32. van Nieuwenhuijze AE, van Lopik T, Smeenk RJ, Aarden LA (2003) Time between onset of apoptosis and release of nucleosomes from apoptotic cells: putative implications for systemic lupus erythematosus. Ann Rheum Dis 62(1):10-14 
33. Zeerleder S, Zwart B, te Velthuis H, Manoe R, Bulder I, Rensink I, Aarden LA (2007) A plasma nucleosome releasing factor (NRF) with serine protease activity is instrumental in removal of nucleosomes from secondary necrotic cells. FEBS Lett 581(28):5382-5388

34. DeShazer D (2004) Genomic diversity of Burkholderia pseudomallei clinical isolates: subtractive hybridization reveals a Burkholderia mallei-specific prophage in B. pseudomallei 1026b. J Bacteriol 186(12):3938-3950

35. Pilsczek FH, Salina D, Poon KK, Fahey C, Yipp BG, Sibley CD, Robbins SM, Green FH, Surette MG, Sugai M, Bowden MG, Hussain M, Zhang K, Kubes P (2010) A novel mechanism of rapid nuclear neutrophil extracellular trap formation in response to Staphylococcus aureus. J Immunol 185(12):7413-7425

36. Hoogendijk AJ, Roelofs JJ, Duitman J, van Lieshout MH, Blok DC, van der Poll T, Wieland CW (2012) R-roscovitine reduces lung inflammation induced by lipoteichoic acid and Streptococcus pneumoniae. Mol Med 18:1086-1095

37. de Boer JH, Hack CE, Verhoeven AJ, Baarsma GS, de Jong PT, Rademakers AJ, de Vries-Knoppert WA, Rothova A, Kijlstra A (1993) Chemoattractant and neutrophil degranulation activities related to interleukin-8 in vitreous fluid in uveitis and vitreoretinal disorders. Invest Ophthalmol Vis Sci 34(12):3376-3385

38. Zeerleder S, Zwart B, Wuillemin WA, Aarden LA, Groeneveld AB, Caliezi C, van Nieuwenhuijze AE, van Mierlo GJ, Eerenberg AJ, Lammle B, Hack CE (2003) Elevated nucleosome levels in systemic inflammation and sepsis. Crit Care Med 31(7):1947-1951

39. Wiersinga WJ, de Vos AF, Wieland CW, Leendertse M, Roelofs JJ, van der Poll T (2008) CD14 impairs host defense against gram-negative sepsis caused by Burkholderia pseudomallei in mice. J Infect Dis 198(9):1388-1397

40. Wiersinga WJ, de Vos AF, de Beer R, Wieland CW, Roelofs JJ, Woods DE, van der Poll T (2008) Inflammation patterns induced by different Burkholderia species in mice. Cell Microbiol 10(1):81-87

41. Wiersinga WJ, Wieland CW, Dessing MC, Chantratita N, Cheng AC, Limmathurotsakul D, Chierakul W, Leendertse M, Florquin S, de Vos AF, White N, Dondorp AM, Day NP, Peacock SJ, van der Poll T (2007) Toll-like receptor 2 impairs host defense in gram-negative sepsis caused by Burkholderia pseudomallei (melioidosis). PLoS Med 4(7):e248

42. Wiersinga WJ, Wieland CW, Roelofs JJ, van der Poll T (2008) MyD88 dependent signaling contributes to protective host defense against Burkholderia pseudomallei. PLoS One 3(10):e3494

43. Thomas GM, Carbo C, Curtis BR, Martinod K, Mazo IB, Schatzberg D, Cifuni SM, Fuchs TA, von Andrian UH, Hartwig JH, Aster RH, Wagner DD (2012) Extracellular DNA traps are associated with the pathogenesis of TRALI in humans and mice. Blood 119(26):6335-6343

44. Yipp BG, Petri B, Salina D, Jenne CN, Scott BN, Zbytnuik LD, Pittman K, Asaduzzaman M, Wu K, Meijndert HC, Malawista SE, de Boisfleury Chevance A, Zhang K, Conly J, Kubes P (2012) Infection-induced NETosis is a dynamic process involving neutrophil multitasking in vivo. Nat Med 18(9):1386-1393

45. Strieter RM, Belperio JA, Keane MP (2002) Cytokines in innate host defense in the lung. J Clin Invest 109(6):699-705

46. Riyapa D, Buddhisa S, Korbsrisate S, Cuccui J, Wren BW, Stevens MP, Ato M, Lertmemongkolchai G (2012) Neutrophil extracellular traps exhibit antibacterial activity against Burkholderia pseudomallei and are influenced by bacterial and host factors. Infect Immun 80(11):3921-3929

47. Xu J, Zhang X, Pelayo R, Monestier M, Ammollo CT, Semeraro F, Taylor FB, Esmon NL, Lupu F, Esmon CT (2009) Extracellular histones are major mediators of death in sepsis. Nat Med 15(11):1318-1321

48. Kager LM, Wiersinga WJ, Roelofs JJ, Meijers JC, Zeerleder SS, Esmon CT, van't Veer C, van der Poll T (2013) Endogenous protein C has a protective role during Gram-negative pneumosepsis (melioidosis). J Thromb Haemost 11(2):282-292

49. Zeerleder S, Stephan F, Emonts M, de Kleijn ED, Esmon CT, Varadi K, Hack CE, Hazelzet JA (2012) Circulating nucleosomes and severity of illness in children suffering from meningococcal sepsis treated with protein C. Crit Care Med 40(12):3224-3229

50. Juneau RA, Pang B, Weimer KE, Armbruster CE, Swords WE (2011) Nontypeable Haemophilus influenzae initiates formation of neutrophil extracellular traps. Infect Immun 79(1):431-438

51. Wiersinga WJ, van der Poll T, White NJ, Day NP, Peacock SJ (2006) Melioidosis: insights into the pathogenicity of Burkholderia pseudomallei. Nat Rev Microbiol 4(4):272-282

52. Thammavongsa V, Missiakas DM, Schneewind O (2013) Staphylococcus aureus degrades neutrophil extracellular traps to promote immune cell death. Science 342(6160):863-866

53. Clark SR, Ma AC, Tavener SA, McDonald B, Goodarzi Z, Kelly MM, Patel KD, Chakrabarti S, McAvoy E, Sinclair GD, Keys EM, Allen-Vercoe E, Devinney R, Doig CJ, Green FH, Kubes P (2007) Platelet TLR4 activates neutrophil extracellular traps to ensnare bacteria in septic blood. Nat Med 13(4):463-469

54. Chanchamroen S, Kewcharoenwong C, Susaengrat W, Ato M, Lertmemongkolchai G (2009) Human polymorphonuclear neutrophil responses to Burkholderia pseudomallei in healthy and diabetic subjects. Infect Immun 77(1):456-463

55. van Zoelen MA, Vogl T, Foell D, Van Veen SQ, van Till JW, Florquin S, Tanck MW, Wittebole X, Laterre PF, Boermeester MA, Roth J, van der Poll T (2009) Expression and role of myeloid-related protein-14 in clinical and experimental sepsis. Am J Respir Crit Care Med 180(11):1098-1106

doi:10.1186/s40635-014-0021-2

Cite this article as: de Jong et al: Neutrophil extracellular traps in the host defense against sepsis induced by

Burkholderia pseudomallei (melioidosis). Intensive Care Medicine Experimental 2014 2:21. 